\title{
In-Situ TEM Investigation of Interactions between Irradiation Defects and Crystal Defects in Austenitic Stainless Steels
}

\author{
Bai Cui ${ }^{1,2}$, Fei Wang ${ }^{1,2}$ and Qiaofeng $\mathrm{Lu}^{1,2}$
}

1. Department of Mechanical \& Materials Engineering, University of Nebraska-Lincoln, Lincoln, NE 68588, USA

2. Nebraska Center for Materials and Nanoscience, University of Nebraska-Lincoln, Lincoln, NE, 68588, USA

This paper presents our recent efforts that use in-situ irradiation TEM experiments as a tool to understand the interaction process of irradiation defects with crystal defects in austenitic stainless steels (ASS). Various irradiation defect clusters, such as dislocation loops, stacking fault tetrahedra, and voids, can form during the heavy-ion irradiation of ASS. [1] Once nucleated, they interact with the pre-existing crystal defects in the ASS, such as dislocations, grain boundaries and interfaces. These interactions may result in the annihilation of irradiation defects, and thus are important for designing the microstructure of materials with improved irradiation resistance. However, the fundamental mechanisms that control the interactions between irradiation defects and crystal defects are not well understood, due to the complexity of the interaction process. Time-resolved in-situ TEM experiments have the ability to offer real-time observation of the development of irradiation defects and their interactions with crystal defects. These in-situ TEM studies provide experimental evidences as the input to aid the development of physical models for the predication of the microstructure - property relationship of ASS in the nuclear reactor environment.

In the first study, a laser shock peening (LSP) process is used to generate significant plastic deformation in 304 ASS, resulting in a high density of deformation twins, stacking faults, and dislocations in the subsurface microstructure (typically $1 \mathrm{~mm}$ in depth). During LSP, the laser-driven shock waves propagate in the ASS and generate a shock-induced pressure of several GPa. If the shock pressure exceeds the dynamic yield strength of ASS, it will experience an extremely high strain-rate $\left(10^{6}-10^{8} \mathrm{~s}^{-1}\right)$ and will be dynamically yielded. In order to understand the effect of LSP on irradiation resistance of ASS, in-situ TEM irradiation experiments have been performed on LSP-treated 304 steels at the IVEM-Tandem facility at Argonne National Laboratory. During $1 \mathrm{MeV} \mathrm{Kr}^{+}$irradiation, twin boundaries and dislocations in LSP-treated 304 steels were observed to serve as effective sinks for the annihilation of irradiation defects. Figure 1 shows an example of the process in which an irradiation defect cluster was interacting with a twin boundary and then gradually annihilated by it. As a result of this annihilation, the irradiation defect density in LSP-treated 304 ASS becomes much reduced compared with the untreated 304 ASS. The implication of this work is that the irradiation resistance of ASS can be improved by an LSP process that generates a high density of irradiation defect sinks such as dislocations, grain boundaries and interfaces in the near-surface microstructure.

In the second study, the micromechanics of dislocation-grain boundary interactions in irradiated ASS is investigated, which has been suggested to establish the conditions necessary for the initiation of irradiation-assisted stress corrosion cracking (IASCC). [2] It is found that cracking susceptibility is higher at the intersection of dislocation channel and grain boundary where slip is discontinuous at the boundary. Recently studies have suggested that the slip transmission criteria, which are derived from unirradiated FCC metals, apply in irradiated FCC metals with the caveat that the importance of the 
resolved shear stress is increased. The resolved shear stress must be sufficient to enable propagation of dislocations through the irradiation defect field. [3] To study the dislocation-grain boundary interaction process in irradiated ASS, in-situ TEM deformation experiments have been performed on irradiated 304 ASS samples. The samples were irradiated by $\mathrm{Kr}^{+}$to a dose of $2.25 \times 10^{18}$ ions $\mathrm{m}^{-2}$, which is equivalent to 1 displacement per atom (dpa). Figure 2 shows the interaction of glissile dislocations on a slip system of $\frac{a}{2}\left[011^{-}\right](111)$ with a $[101] / 56^{\circ}$ grain boundary. These dislocations pile up on this grain boundary (Figure 2a), but the slip transmission did not occur. No outgoing dislocations were observed to emit from this grain boundary into the neighboring grain (Figure $2 \mathrm{~b}$ ). This is explained as that the conditions favorable for slip transmission are not satisfied. Due to the presence of irradiation damage, the local resolved shear stress was not sufficient to enable emission of dislocations from the grain boundary. [4]

\section{References:}

[1] S. Zinkle, P. Maziasz and R. Stoller, Journal of Nuclear Materials 206 (1993) p. 266.

[2] B. Cui et al, Philosophical Magazine 94 (2014) p. 4197.

[3] B. Cui et al, Acta Materialia 65 (2014) p. 150.

[4] The authors acknowledge funding from the Nebraska Center for Energy Sciences Research. The authors thank Mark Kirk and Meimei Li at Argonne National Laboratory for help with the operation of the IVEM-Tandem Facility.
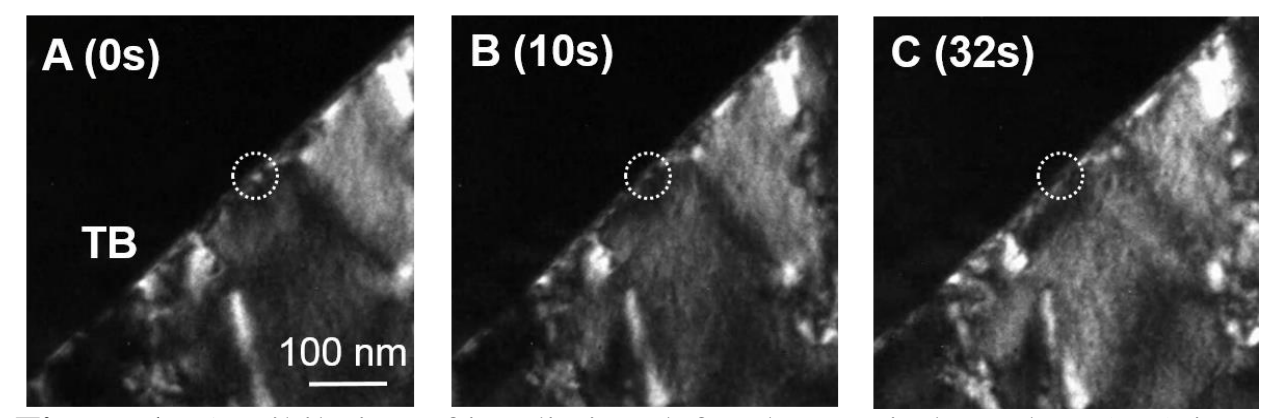

Figure 1. Annihilation of irradiation defect by a twin boundary (TB) in a LSP-treated 304 steel sample. The snapshots show an irradiation defect cluster (bright spot in the circle): (A) formed in the vicinity of a TB; (B) partially annihilated; and (C) completely annihilated by this TB.
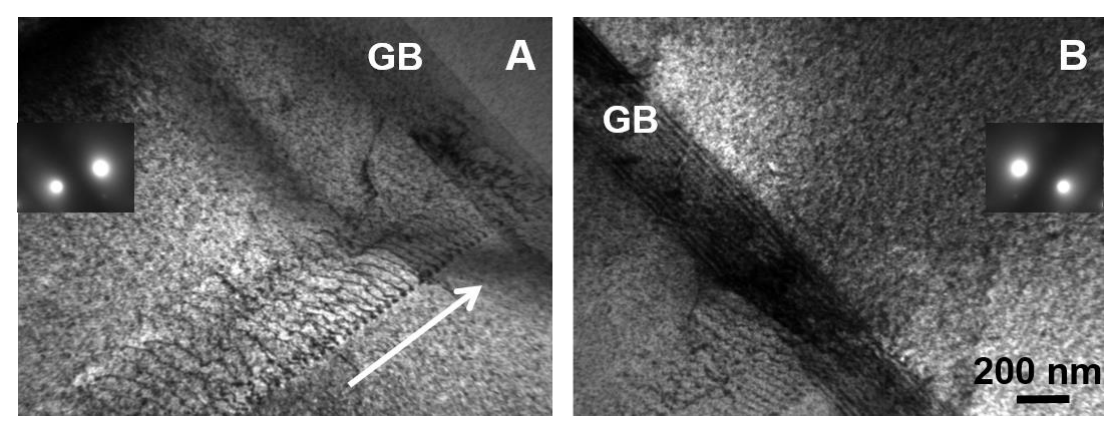

Figure 2. Accommodation of dislocations by a [101]/56 ${ }^{\circ}$ grain boundary (GB) in $1 \mathrm{dpa} \mathrm{Kr}^{+}$-irradiated 304 steel. (A) An array of dislocations piles up on the grain boundary in the left grain. The motion direction of dislocations is indicated by the white arrow. (B) No outgoing dislocations emit from this grain boundary into the right grain. Diffraction patterns are inserted to show different diffraction conditions used for each micrograph. See the text for details. 